\title{
Caracterização vegetacional do Monumento Natural Vale dos Dinossauros em Sousa,
}

\section{Paraíba, Brasil}

Vegetational characterization of the Natural Monument Vale dos Dinosauros in Sousa Paraíba, Brazil

Caracterización vegetacional del Monumento Natural Vale dos Dinosauros en Sousa Paraíba, Brasil

\section{Resumo}

O objetivo do presente trabalho foi caracterizar a vegetação arbórea da mata ciliar do trecho do Rio do Peixe, inserido no Monumento Natural do Vale dos Dinossauros (MONA), em Sousa-PB. Foram mensuradas árvores em 20 unidades amostrais quadradas de $400 \mathrm{~m}^{2}$, dispostas às margens do Rio do Peixe, de maneira equidistante. Foram mensuradas, com fita métrica, a Circunferência do fuste a $1,30 \mathrm{~m}$ do solo e a $0,3 \mathrm{~m}$ do solo dos indivíduos arbóreos com circunferência a altura do peito $(1,3 \mathrm{~m})$ maior ou igual a $6 \mathrm{~cm}$, além da altura total dos indivíduos, com o auxílio de vara graduada. Avaliou-se a diversidade, equabilidade, florística e as estruturas horizontal e vertical, além da distribuição diamétrica. Foram identificados 919 indivíduos, 16 famílias botânicas, 32 gêneros e 35 espécies. As famílias botânicas mais representativas em número de indivíduos foram a Fabaceae Linl. a Combretaceae R. Br., sendo a Fabaceae a mais representativa em número de espécies. A área apresenta boa diversidade florística, com boa distribuição dos indivíduos nas espécies. A espécie com maior importância e abundância na área foi o Combretum leprosum Mart. (mofumbo), com 32\% dos indivíduos amostrados, e 16,69\% do valor de importância. As espécies Cenostigma pyramidale (Tul.) Gagnon \& G.P.Lewis., Senegalia bahiensis (Tul.) Gagnon \& G.P.Lewis e Pseudobombax marginatum (A.St.-Hil., Juss. \& Cambess.) A. Robyns, foram menos abundantes com um indivíduo cada, em toda a amostra. A distribuição diamétrica apresentou comportamento característico de populações naturais, em J-invertido. Há forte presença de espécies exóticas na área, porém, na amostra, constatou-se a presença de Prosopis juliflora (Sw) DC., Leucaena leucocephala (Lam.) de Wit., Cryptostegia madagascariensis Bojer. e Malpighia glabra L., espécies que naturalmente não deveriam existir em unidade de conservação.

Palavras-chave: Diversidade; Conservação; Mata ciliar; Semiárido brasileiro.

\begin{abstract}
The objective of the present work was to characterize the arboreal vegetation of the riparian forest of Rio do Peixe, in the Natural Monument of Vale dos Dinossauros (MONA), in Sousa-PB. Trees were measured in 20 square sampling units of $400 \mathrm{~m}^{2}$, arranged on the banks of the Rio do Peixe, in an equidistant manner. The circumference of the fuste at $1.30 \mathrm{~m}$ from the ground and at $0.3 \mathrm{~m}$ from the ground of the arboreal individuals with circumference at breast height $(1.3 \mathrm{~m})$ greater than or equal to $6 \mathrm{~cm}$ were measured with a measuring tape, in addition to the total height of the individuals, with the aid of graduated rod. Were evaluated floristic, diversity, evenness, and horizontal and vertical
\end{abstract}


structures, in addition to diametric distribution. Were identified 919 individuals, 16 botanical families, 32 genera and 35 species. The most representative botanical families in number of individuals were the Fabaceae Linl. a Combretaceae R. Br., Fabaceae being the most representative in number of species. The area presents good floristic diversity and good distribution of individuals per species. The specie more importance and abundance in the area was Combretum leprosum Mart. (mofumbo) with $32 \%$ of the individuals sampled and $16.69 \%$ of the importance value. The species Cenostigma pyramidale (Tul.) Gagnon \& G.P.Lewis., Senegalia bahiensis (Tul.) Gagnon \& G.P.Lewis e Pseudobombax marginatum (A.St.-Hil., Juss. \& Cambess.) A. Robyns were less abundant, with one individual each. Diametric distribution showed behavior characteristic of natural populations, in inverted-J. There is a strong presence of exotic species in the area, but in was found the sample, the presence of Prosopis juliflora (Sw) DC., Leucaena leucocephala (Lam.) de Wit., Cryptostegia madagascariensis Bojer. e Malpighia glabra L., species that naturally should not exist in the area.

Keywords: Diversity; Conservation; Riparian forest; Brazilian semiarid.

\section{Resumen}

El objetivo del presente trabajo fue caracterizar la vegetación arbórea del bosque de ribera del tramo Rio do Peixe, en el Monumento Natural de Vale dos Dinossauros (MONA), en Sousa-PB. Los árboles se midieron en 20 unidades muestra cuadradas de $400 \mathrm{~m}^{2}$, dispuestas del Rio do Peixe, de manera sistematica. La circunferencia del tronco a 1,30 $\mathrm{m}$ del suelo y a $0,3 \mathrm{~m}$ del suelo, con una cinta métrica de los individuos arbóreos con circunferencia a la altura del pecho $(1,3 \mathrm{~m})$ mayor o igual a $6 \mathrm{~cm}$, además de la altura con la ayuda de una varilla graduada. Se evaluó la florísticas, diversidad, uniformidad, estructuras y horizontales y verticales, así como la distribución diametral. Se identificaron 919 individuos, 16 familias botánicas, 32 géneros y 35 especies. Las familias botánicas más representativas en número de individuos fueron las Fabaceae Linl. a Combretaceae R. Br., siendo las Fabaceae las más representativas en número de especies. El área presenta buena diversidad florística y buena distribución de individuos en especies. La especie con mayor importancia y abundancia en el área fue Combretum leprosum Mart. (mofumbo) con 32\% de los individuos muestreados y $16.69 \%$ del valor de importancia. Las especies Cenostigma pyramidale (Tul.) Gagnon \& G.P.Lewis., Senegalia bahiensis (Tul.) Gagnon \& G.P.Lewis e Pseudobombax marginatum (A.St.-Hil., Juss. \& Cambess.) A. Robyns fueron menos abundantes, con un individuo cada una. La distribución diamétrica mostró un comportamiento característico de las poblaciones naturales, en $\mathbf{J}$ invertida. Hay una fuerte presencia de especies exóticas en la zona, ser muestreado Prosopis juliflora (Sw) DC., Leucaena leucocephala (Lam.) de Wit., Cryptostegia madagascariensis Bojer. e Malpighia glabra L., especies que naturalmente no deberían existir en la zona.

Palabras clave: Diversidad; Conservación; Bosque ciliar; Semiárido brasileño.

\section{Introdução}

Compreendendo cerca de $11 \%$ do território nacional, a caatinga está presente na maior parte dos estados do Piauí, Ceará, Paraíba, Pernambuco, Alagoas, Rio Grande do Norte, Sergipe, Bahia e a parte nordeste de Minas Gerais (Giulietti et al., 2004; Leal et al., 2005). A caatinga é composta por diversificadas paisagens, tipos e comportamentos vegetacionais, características influenciadas pelas condições climáticas, edáficas e pela própria ação humana.

Esta vegetação é constantemente explorada para atender às demandas locais e regionais, agravando-se por predominar a exploração ilegal e desordenada. Moreira (2013) menciona que, num período de seis anos (2002 a 2008), a caatinga perdeu cerca de 16.600 quilômetros quadrados de vegetação predominante nativa, o que corresponde a aproximadamente $2 \%$ de todo o bioma. O processo de desmatamento não estagnou, haja visto que, de 2019 para 2020, a caatinga perdeu 49.220 ha para o desmatamento, de acordo com dados do Relatório Anual de Desmatamento no Brasil (Azevedo et al, 2020). É um dos biomas mais ameaçados do Brasil, em consequência do uso inadequado e insustentável dos recursos naturais oferecidos, além do agravante de ser o bioma com a menor área preservada sob unidades de conservação (Rocha, 2011; Silva et al., 2021).

Fernandes e Medeiros (2009) ressaltam que a desertificação na região nordestina está levando à redução da produtividade das terras, ocasionando uma perda na renda das famílias e fazendo com que cada vez mais se desloquem para grandes centros urbanos. Os autores ainda mencionam que, para diminuir os avanços desse processo, é preciso tomar medidas sociais e tecnológicas com urgência.

Nesse sentido, Dias (2001) cita que uma das áreas mais exploradas e degradadas que precisam ser conservadas do bioma caatinga são as matas ciliares, que legalmente são consideradas Áreas de Preservação Permanentes (APP). Na caatinga, 
preservar a mata ciliar é vital para a vida dos rios principais e seus afluentes, considerando principalmente que a estação da seca pode durar alguns anos.

A maior parte dos rios dessa região são efêmeros, e o escoamento da água só ocorre durante ou imediatamente após as chuvas. E, devido à baixa pluviosidade em algumas regiões do bioma, o assoreamento, poluição dos rios, entre outros, aceleram a degradação das matas ciliares e, consequentemente, há uma má qualidade da água que subsiste nos rios (Lima, 2008).

Em outra frente de tentativa de frear o problema, o MMA, juntamente com outros órgãos, vem criando Unidades de Conservações (UC) no bioma para que, em médio e longo prazos, esses níveis de desmatamentos diminuam. Entretanto, o Bioma Caatinga conta com apenas 213 UC's (8,99\%) da sua área protegida, sendo 60 (2,4\%) de proteção integral (CNUC, 2020).

Ramos et al. (2020) relatam que o desconhecimento sobre as condições da vegetação ciliar dos rios contribui para não frear ou reduzir as atividades realizadas de forma inadequadas de uso desta vegetação, reduzindo a ocorrência de importantes espécies nas áreas ciliares. Reforçam, ainda, que os problemas citados são ocorrentes na Paraíba, especificamente pelo registro dos altos níveis de degradação nas bacias e sub-bacias hidrográficas.

Estudos sobre a composição florística e fitossociológica são essenciais para a conservação dos recursos naturais, pois, através do conhecimento do potencial florestal, é possível compreender a sua estrutura e diversidade, as quais são fatores importantes para o estabelecimento do manejo sustentável. Segundo Chaves et al. (2013), esses estudos são uma ferramenta fundamental no auxílio da análise da necessidade de medidas direcionadas à preservação e ao uso dos recursos florestais.

O Brasil, por exemplo, possui uma série de unidades de conservação - UC's, as quais são reguladas pela Lei no 9.985, de 18 de julho de 2000, que instituiu o Sistema Nacional de Unidades de Conservação - SNUC (Brasil, 2000). As UC's são classificadas em duas categorias: as de proteção integral e as de uso sustentável. Dentre as de proteção integral, encontram-se os monumentos naturais.

Na Paraíba, existe, na região geográfica intermediária de Sousa-Cajazeiras (IBGE, 2017), a Unidade de conservação Estadual, o Monumento Natural Vales dos Dinossauros, localizado no município de Sousa. Essa UC foi criada com o objetivo de preservar as pegadas de dinossauros encontradas no lugar. Complementa-se ao objetivo da UC, conservar os recursos naturais presentes nas áreas, como solo, água e vegetação, bem como suas inter-relações. Desta forma, objetiva-se caracterizar a vegetação arbórea da mata ciliar de um trecho do Rio do Peixe, inserido no Monumento Natural do Vale dos Dinossauros em Sousa-PB.

\section{Metodologia}

A área da pesquisa realizada consiste no Monumento Natural do Vale dos Dinossauros, Sousa - PB (MONA Vale dos Dinossauros), o qual foi criado pelo Decreto n. ${ }^{\circ}$ 23.832, de 27 de dezembro de 2002. A área do MONA é de 38,8 hectares (Paraíba, 2002). Observa-se, no local, infraestrutura de apoio aos visitantes (recepção e museu de exposição), passarelas e a obra de maior destaque é o canal de alívio, concebido para desviar parte da água do Rio do Peixe, durante o período chuvoso, para propiciar maior preservação das pegadas. A UC está localizada a aproximadamente $6 \mathrm{~km}$ da cidade de Sousa, cujas coordenadas geográficas da sede são: $6^{\circ} 43^{\prime} 56^{\prime \prime}$ latitude sul e 38 $15^{\circ}$ ' 42” longitude oeste. O clima, segundo a classificação de Köppen, é semiárido - BSh. A temperatura média mensal é superior a $18^{\circ} \mathrm{C}$, ventos predominando entre SE e NE (Alvares et al. 2013).

A amostra contou com 20 unidades amostrais quadradas de $400 \mathrm{~m}^{2}(20 \mathrm{~m} \times 20 \mathrm{~m})$, dispostas de maneira equidistante às margens do Rio do Peixe, em ambas as laterais e na área de margem do canal de alívio do MONA. Todos os procedimentos amostrais de coleta de dados foram realizados de acordo com o Protocolo de Medições de Parcelas Permanentes da Rede de 
Manejo Florestal da Caatinga (RMFC, 2005), em que foram mensuradas, com fita métrica, a Circunferência a Altura do Peito (CAP) a 1,30 m do solo e a Circunferência na Base (CNB) a 0,3 m do solo dos indivíduos arbóreos com CAP igual ou superior a $6 \mathrm{~cm}(\mathrm{CAP} \geq 6 \mathrm{~cm})$, o que equivale a um diâmetro (DAP) mínimo de $1,9 \mathrm{~cm}$, além da altura total $(\mathrm{H})$ dos indivíduos, com o auxílio de uma vara graduada. Para distribuição diamétrica, os indivíduos que apresentaram bifurcação, ou seja, mais de um DAP por indivíduo, tiveram os dados ajustados para o diâmetro equivalente $\left(D_{e q}\right)$, conforme equação 1.

$D_{e q}=\sqrt{\sum_{i=1}^{n} D A P_{i}^{2}}$

Em que: $\mathrm{DAP}=(D A P=C A P / \pi)=$ diâmetro a altura do peito $(1,3 \mathrm{~m}$ do solo $)$;

A composição florística foi listada com base na distribuição dos indivíduos em espécies e famílias, e a diversidade e equabilidade com os índices de Shannon-Wiener (H’) e de Pielou (J'), respectivamente, além da dominância de Simpson (C). Os parâmetros avaliados da estrutura horizontal foram a densidade absoluta e relativa, a frequência absoluta e relativa, a dominância absoluta e relativa, índice de valor de importância e estrutura diamétrica. Além da estrutura vertical, a determinação dos estratos foi realizada por divisão em estratos Inferior (1), Médio (2) e, Superior (3), através da frequência relativa das alturas (Longhi, 1980; citado por Souza \& Soares, 2013).

Os dados foram coletados em 2019, oportunizado pelo período de disponibilidade de material vegetativo para identificação das espécies em campo, comparando-as com a literatura e sendo coletado material botânico para devida identificação, sendo as espécies posteriormente encaminhadas ao Herbário "Rita Baltazar de Lima", pertencente à Universidade Federal de Campina Grande (UFCG), no Centro de Saúde e Tecnologia Rural (CSTR). A lista das espécies seguiu a classificação do Angiosperm Phylogeny Group (APG IV, 2016), e a terminologia das espécies e seus respectivos autores estão descritos conforme Lista de Espécies da Flora do Brasil (2020). Já os nomes populares estão descritos conforme o conhecimento local.

\section{Resultados e Discussão}

A representatividade da área foi atendida, considerando que a curva de acumulação de espécies determinou a suficiência com 16 unidades amostrais, conforme pode ser verificado na Figura 1, o que implica que o levantamento é representativo da população inventariada. 
Figura 1. Curva de acumulação de espécies arbóreas amostradas no MONA Vale dos Dinossauros, Sousa-PB.

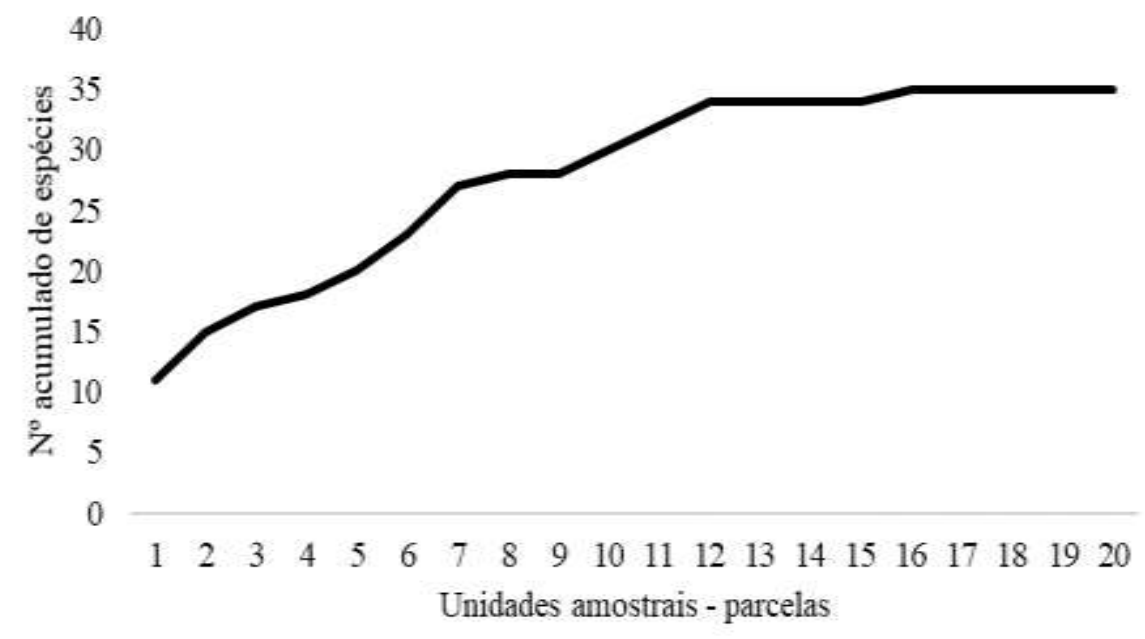

Fonte: Autores (2021).

No levantamento florístico (Tabela 1), considerando todas as parcelas, foram identificados 919 indivíduos (1552 fustes - DAP's), distribuídos em 16 famílias botânicas, 32 gêneros e 35 espécies, sendo as espécies Cenostigma pyramidale (Tul.) Gagnon \& G.P.Lewis. Senegalia bahiensis (Benth.) Seigler \& Ebinger e Pseudobombax marginatum (A.St.-Hil., Juss. \& Cambess.) A. Robyns, representadas por apenas um indivíduo cada, em toda a amostra. A espécie com maior abundância foi o Combretum leprosum Mart. (Mofumbo), com 298 indivíduos dos 919 amostrados. 
Tabela 1. Composição florística dos indivíduos identificados no MONA Vale dos Dinossauros, Sousa-PB.

\begin{tabular}{|c|c|c|c|}
\hline Família & Nome Científico & Nome comum & $\begin{array}{l}\text { Número de } \\
\text { indivíduos }\end{array}$ \\
\hline \multirow[t]{15}{*}{ Fabaceae } & Prosopis juliflora (Sw) DC. & Algaroba & 13 \\
\hline & Anadenanthera colubrina (Vell.) Brenan. & Angico Vermelho & 5 \\
\hline & Cenostigma pyramidale (Tul.) Gagnon \& G.P.Lewis. & Catingueira & 1 \\
\hline & Senegalia bahiensis (Benth.) Seigler \& Ebinger & Espinheiro branco & 1 \\
\hline & Senegalia polyphylla (DC.) Britton \& Rose & Espinheiro preto & 24 \\
\hline & Longocarpus seriseus (Poir.) Kunth ex DC. & Ingá & 10 \\
\hline & Libidibia ferrea (Mart. ex Tul.) L.P.Queiroz. & Jucá & 19 \\
\hline & Piptadenia retusa (Jacq.) P.G.Ribeiro, Seigler \& Ebinger. & Jurema Branca & 50 \\
\hline & Mimosa tenuiflora (Willd.) Poir. & Jurema Preta & 29 \\
\hline & Leucaena leucocephala (Lam.) de Wit. & Leucena & 6 \\
\hline & Geoffroea spinosa Jacq. & Marizeiro & 97 \\
\hline & Bauhinia forficata Link. & Mororó & 5 \\
\hline & Senna sp. & - & 2 \\
\hline & Enterolobium contortisiliquum (Vell.) Morong. & Tamboril & 33 \\
\hline & Mimosa arenosa (Willd.) Poir. & Unha de Gato & 8 \\
\hline \multirow[t]{2}{*}{ Combretaceae } & Combretum sp. & - & 3 \\
\hline & Combretum leprosum Mart. & Mofumbo & 298 \\
\hline \multirow[t]{2}{*}{ Euphorbiaceae } & Jatropha mollissima (Pohl) Baill. & Pinhão bravo & 28 \\
\hline & Croton conduplicatus Kunth. & Quebra Faca & 62 \\
\hline Capparaceae & Cynophalla flexuosa (L.) J.Presl & Feijão bravo & 60 \\
\hline Polygoniaceae & Triplaris gardneriana Wedd. & Cauaçu & 36 \\
\hline \multirow[t]{2}{*}{ Anacardiaceae } & Astronium urundeuva (M.Allemão) Engl & Aroeira & 27 \\
\hline & Spondias mombin L. & Cajá & 6 \\
\hline \multirow[t]{2}{*}{ Erythroxylaceae } & Erythroxylum sp. & - & 30 \\
\hline & Erythroxylum pungens O.E.Schulz & Rompe gibão & 2 \\
\hline Rhamnaceae & Sarcomphalus joazeiro (Mart.) Hauenshild. & Juazeiro & 16 \\
\hline \multirow[t]{2}{*}{ Apocynaceae } & Cryptostegia madagascariensis Bojer. & Boca de leão & 7 \\
\hline & Aspidosperma pyrifolium Mart. \& Zucc. & Pereiro & 7 \\
\hline Sapotaceae & Sideroxylon obtusifolium (Humb. ex Roem. \& Schult.) T. D. Penn. & Quixabeira & 14 \\
\hline Bignoniaceae & Handroanthus impetiginosus (Mart. ex DC.) Mattos. & Ipê Roxo & 11 \\
\hline Chysobalanaceae & Microdesmia rigida (Benth.) Sothers \& Prance & Oiticica & 2 \\
\hline Malpighiaceae & Malpighia glabra L. & Acerola & 2 \\
\hline Rubiaceae & Genipa americana L. & Jenipapo & 2 \\
\hline Sapindaceae & Sapindus saponaria $\mathrm{L}$ & Saboneteira & 2 \\
\hline Malvaceae & Pseudobombax marginatum (A.St.-Hil., Juss. \& Cambess.) A. Robyns & Embiratanha & 1 \\
\hline
\end{tabular}

Fonte: Autores (2021).

A família mais representativa em riqueza de espécies foi a Fabaceae, com 15 espécies, consequentemente em número de indivíduos (303); a segunda família mais representativa em número de indivíduos foi a Combretaceae, com 301 indivíduos amostrados, enfatizando a grande influência do C. leprosum, com 298 indivíduos. Das 16 famílias botânicas representadas, além da Fabaceae, 3 famílias apresentaram dois gêneros, e as demais (12 famílias) apresentaram apenas um gênero. Outros trabalhos realizados na caatinga também identificaram a Fabaceae como a família botânica mais representativa nas áreas estudadas, como nos trabalhos de Souza e Medeiros (2013); Medeiros et al. (2018); Justino et al. (2019); Lima et al. (2019) e Medeiros et al. (2021).

Quanto ao número de espécies e famílias botânicas registradas neste trabalho, foi menor que o registrado em outros levantamentos de mata ciliar na caatinga, como nos trabalhos de Silva, et al. (2015), com 105 espécies e 44 famílias, Farias et al. (2017), que identificaram 57 espécies e 22 famílias, e Ramos et al. (2020), que registraram 107 e 37, espécies e famílias, respectivamente. Neste trabalho, consta um agravante da degradação das matas ciliares pela presença de muitas espécies invasoras.

A diversidade da área foi representada pelo índice de Shannon-Weaver (H'), com 2,63 nats ind ${ }^{-1}$, e a uniformidade de distribuição das espécies foi aferida pelo índice de Pielou (J'), com 0,74, expressando boa diversidade, confirmada pelo índice de Simpson (C) de 0,93. Isso implica dizer que a área apresenta boa diversidade e relativa homogeneidade, mesmo com uma espécie que apresenta alta dominância, como é o caso do C. leprosum, que supera os 32\% do número de indivíduos 
amostrados. Os dados deste estudo são superiores aos constatados por Batista et al. (2019), que obtiveram dados de 0,97 para H', 0,44 para J', e C de 0,44 em área de caatinga. No entanto, os valores encontrados neste estudo foram inferiores aos de outros trabalhos realizados em outras áreas de caatinga, como os obtidos por Silva, L. et al. (2015), tendo o índice de Shannon de 3,32 nats.ind ${ }^{-1} \mathrm{em}$ área conservada de mata ciliar.

Quanto à caracterização fitossociológica da área, as espécies de maior valor de importância foram a C. leprosum, G. spinosa, E contorstisiliquum e C. flexuosa, juntas representando 39,26\% do valor de importância das espécies amostradas (Tabela 2).

Tabela 2. Parâmetros fitossociológicos, em ordem decrescente do valor de importância (VI), para as espécies arbustivoarbóreas amostradas identificadas no MONA Vale dos Dinossauros, Sousa-PB.

\begin{tabular}{|c|c|c|c|c|c|c|c|c|}
\hline Nome Científico & DA & DR & FA & FR & DoA & DoR & VI & VI $(\%)$ \\
\hline Combretum leprosum & 372,5 & 32,43 & 85 & 10,69 & 0,799 & 6,94 & 50,057 & 16,69 \\
\hline Geoffroea spinosa & 121,25 & 10,55 & 45 & 5,66 & 1,601 & 13,89 & 30,11 & 10,04 \\
\hline Enterolobium contortisiliquum & 41,25 & 3,59 & 35 & 4,4 & 1,286 & 11,16 & 19,154 & 6,38 \\
\hline Cynophalla flexuosa & 75 & 6,53 & 85 & 10,69 & 0,141 & 1,22 & 18,444 & 6,15 \\
\hline Sarcomphalus joazeiro & 20 & 1,74 & 50 & 6,29 & 1,08 & 9,38 & 17,406 & 5,8 \\
\hline Piptadenia retusa & 62,5 & 5,44 & 60 & 7,55 & 0,241 & 2,09 & 15,077 & 5,03 \\
\hline Triplaris gardneriana & 45 & 3,92 & 45 & 5,66 & 0,575 & 4,99 & 14,566 & 4,86 \\
\hline Croton conduplicatus & 77,5 & 6,75 & 40 & 5,03 & 0,22 & 1,91 & 13,69 & 4,56 \\
\hline Erythroxylum sp. & 37,5 & 3,26 & 20 & 2,52 & 0,816 & 7,08 & 12,861 & 4,29 \\
\hline Prosopis juliflora & 16,25 & 1,41 & 20 & 2,52 & 0,871 & 7,56 & 11,488 & 3,83 \\
\hline Microdesmia rígida & 2,5 & 0,22 & 10 & 1,26 & 1,148 & 9,97 & 11,443 & 3,81 \\
\hline Mimosa tenuiflora & 36,25 & 3,16 & 35 & 4,4 & 0,361 & 3,13 & 10,692 & 3,56 \\
\hline Longocarpus seriseus & 12,5 & 1,09 & 15 & 1,89 & 0,751 & 6,52 & 9,498 & 3,17 \\
\hline Astronium urundeuva & 33,75 & 2,94 & 20 & 2,52 & 0,31 & 2,69 & 8,143 & 2,71 \\
\hline Jatropha molíssima & 35 & 3,05 & 30 & 3,77 & 0,071 & 0,62 & 7,441 & 2,48 \\
\hline Spondias mombin & 7,5 & 0,65 & 5 & 0,63 & 0,404 & 3,5 & 4,785 & 1,6 \\
\hline Libidibia férrea & 23,75 & 2,07 & 15 & 1,89 & 0,057 & 0,5 & 4,453 & 1,48 \\
\hline Senegalia polyphylla & 30 & 2,61 & 10 & 1,26 & 0,059 & 0,51 & 4,379 & 1,46 \\
\hline Handroanthus impetiginosus & 13,75 & 1,2 & 15 & 1,89 & 0,149 & 1,29 & 4,378 & 1,46 \\
\hline Anadenanthera colubrina & 6,25 & 0,54 & 10 & 1,26 & 0,202 & 1,75 & 3,554 & 1,18 \\
\hline Aspidosperma pyrifolium & 8,75 & 0,76 & 20 & 2,52 & 0,022 & 0,19 & 3,467 & 1,16 \\
\hline Sideroxylon obtusifolium & 17,5 & 1,52 & 10 & 1,26 & 0,064 & 0,56 & 3,341 & 1,11 \\
\hline Cryptostegia madagascariensis & 8,75 & 0,76 & 15 & 1,89 & 0,018 & 0,15 & 2,801 & 0,93 \\
\hline Leucaena leucocephala & 7,5 & 0,65 & 15 & 1,89 & 0,008 & 0,07 & 2,609 & 0,87 \\
\hline Mimosa arenosa & 10 & 0,87 & 10 & 1,26 & 0,047 & 0,4 & 2,533 & 0,84 \\
\hline Bauhinia forficata & 6,25 & 0,54 & 15 & 1,89 & 0,008 & 0,07 & 2,502 & 0,83 \\
\hline Pseudobombax marginatum & 1,25 & 0,11 & 5 & 0,63 & 0,138 & 1,2 & 1,94 & 0,65 \\
\hline Genipa americana & 2,5 & 0,22 & 10 & 1,26 & 0,016 & 0,14 & 1,612 & 0,54 \\
\hline Malpighia glabra & 2,5 & 0,22 & 10 & 1,26 & 0,007 & 0,06 & 1,535 & 0,51 \\
\hline Erythroxylum pungens & 2,5 & 0,22 & 10 & 1,26 & 0,003 & 0,03 & 1,503 & 0,5 \\
\hline Sapindus saponária & 2,5 & 0,22 & 5 & 0,63 & 0,028 & 0,24 & 1,087 & 0,36 \\
\hline Combretum sp. & 3,75 & 0,33 & 5 & 0,63 & 0,005 & 0,04 & 0,997 & 0,33 \\
\hline Senna sp. & 2,5 & 0,22 & 5 & 0,63 & 0,004 & 0,03 & 0,878 & 0,29 \\
\hline Senegalia bahiensis & 1,25 & 0,11 & 5 & 0,63 & 0,011 & 0,09 & 0,832 & 0,28 \\
\hline Cenostigma pyramidale & 1,25 & 0,11 & 5 & 0,63 & 0,001 & 0,01 & 0,745 & 0,25 \\
\hline Totais & 1148,75 & 100 & 795 & 100 & 11,519 & 100 & 300 & 100 \\
\hline
\end{tabular}

Em que: $\mathrm{DA}=$ densidade absoluta $\left(\right.$ ind.ha $\left.^{-1}\right), \mathrm{DR}=$ densidade relativa $(\%), \mathrm{FA}=$ frequência absoluta $(\%$ de parcelas onde ocorre a espécie), $\mathrm{FR}=$ frequência relativa $(\%), \mathrm{DoA}=$ dominância absoluta $\left(\mathrm{m}^{2} \cdot \mathrm{ha}^{-1}\right)$ e DoR = dominância relativa $(\%), \mathrm{VI}=$ valor de importância absoluto e $\mathrm{VI}(\%)=$ valor de importância relativo. Fonte: Autores (2021).

Em relação à distribuição diamétrica, as classes de diâmetro foram distribuídas em amplitude de $3 \mathrm{~cm}$, a partir do diâmetro mínimo de inclusão, equivalendo a $1,9 \mathrm{~cm}$, e a última classe com os indivíduos que apresentaram diâmetros superiores a $28,9 \mathrm{~cm}$. Desta forma, todos os indivíduos mensurados foram distribuídos nas respectivas classes de diâmetro, conforme Figura 2. 
Figura 2. Distribuição diamétrica e respectivas áreas basais $\left(\mathrm{m}^{2} \cdot \mathrm{ha}^{-1}\right)$ dos indivíduos amostrados no MONA Vale dos Dinossauros, Sousa-PB.

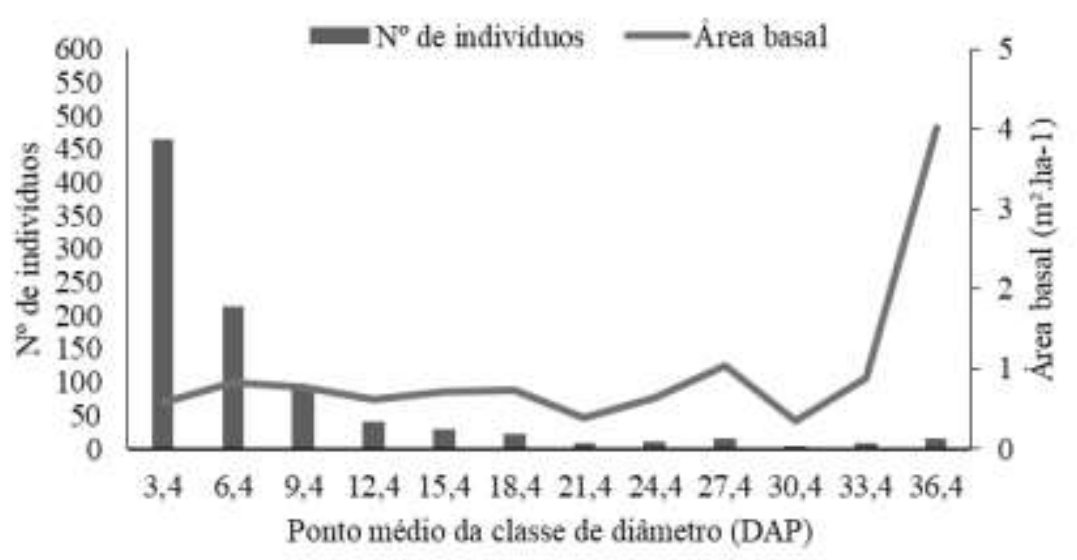

Fonte: Autores (2021).

Os diâmetros variaram de $1,9 \mathrm{~cm}$ a $64 \mathrm{~cm}$, sendo este diâmetro mais expressivo referente a um indivíduo de $S$. joazeiro. A distribuição diamétrica dos indivíduos seguiu o padrão comum de florestas naturais, em J-invertido, apresentando a maior concentração em número de indivíduos nas classes de menor diâmetro. Este comportamento é comum na Caatinga, de acordo com Calixto Junior e Drumond (2011) e reforçado por Guedes et al. (2012), os quais mencionam que tal comportamento é observado, também, em caatinga preservada. Comportamento similar foi verificado também por Medeiros et al. (2018) em caatinga, no município de São Mamede-PB, e Batista et al. (2019) em área de caatinga manejada.

Considerando todos os indivíduos amostrados e a distribuição diamétrica, todos os indivíduos que apresentaram diâmetros superiores aos estabelecidos na última classe (36,4 de ponto médio) contabilizaram mais 14 indivíduos. Em consequência destes diâmetros superiores, esta classe contribuiu com a maior área basal dentre as classes, representando $34,87 \%$ da área basal total estimada.

A área basal total estimada para a área foi de $11,519 \mathrm{~m}^{2} \cdot \mathrm{ha}^{-1}$, valor considerado alto para áreas de caatinga, exceto quando tem influência de reservatórios e mananciais hídricos, pois essa estimativa apresenta valor superior aos geralmente constatados na caatinga, como pode ser verificado nos estudos de Santos et al. (2017) e Medeiros et al. (2018). No entanto, a área basal estimada neste estudo foi inferior à aferida por Marques et al. (2020), em mata ciliar do rio Sucuru, em Coxixola (PB), de 12,71 $\mathrm{m}^{2} \cdot \mathrm{ha}^{-1}$, e por Lacerda e Barbosa (2018), que estimaram área basal de 15,62 $\mathrm{m}^{2} \cdot \mathrm{ha}^{-1}$ na mata ciliar do rio Taperoá, na Paraíba.

Quanto à distribuição dos indivíduos em classes de altura, a maior concentração de indivíduos aconteceu no estrato médio (classe central) (Figura 3). Assim também ocorreu em outros trabalhos realizados na ecorregião, como o de Santos et al. (2017) e Batista et al. (2019). Entretanto, a maior concentração da área basal ocorreu no estrato vertical com as maiores alturas, denotando serem as árvores mais desenvolvidas em altura e diâmetro. 
Figura 3. Distribuição do número de indivíduos e área basal por classe de altura, dos indivíduos amostrados no MONA Vale dos Dinossauros, Sousa-PB.

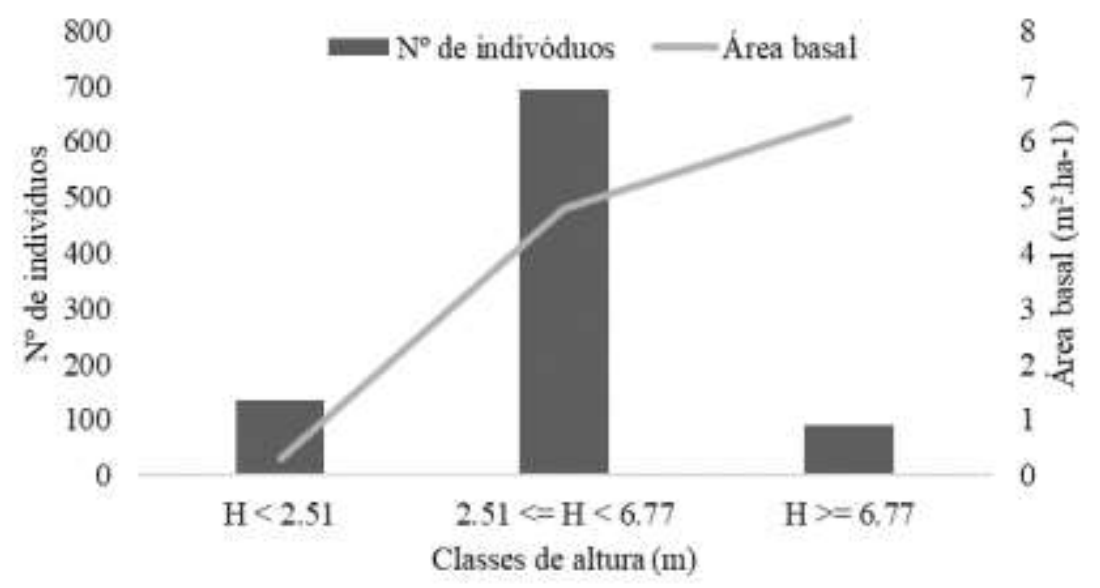

Fonte: Autores (2021).

Considerando apenas as espécies presentes no estrato superior, denotando a capacidade de estas espécies em desenvolverem-se e associando-se as próprias características intraespecíficas, podemos verificar que, dentre as espécies exóticas, constatou-se a presença da Prosopis juliflora no estrato superior, espécie esta que é bastante abrangente na área, no entanto, na amostragem da mata ciliar, foi representada por apenas 13 indivíduos, distribuídos nos três estratos de altura. Quanto às espécies nativas, excetuando as pioneiras que naturalmente apresentam baixo porte, como o Croton conduplicatus, Jatropha mollissima, Bauhinia forficata, dentre outras, não alcançam esta altura e, portanto, não foram amostrados indivíduos na classe superior.

\section{Conclusão}

As famílias botânicas mais representativas em número de indivíduos foram a Fabaceae e Combretaceae, sendo a Fabaceae a mais representativa em número de espécies.

Os índices de diversidade indicaram uma boa diversidade florística, considerando as características da caatinga, com boa distribuição dos indivíduos nas espécies.

Dentre as 35 espécies, a que apresentou a maior importância foi o Combretum leprosum. Como espécies raras na área, tem-se a Cenostigma pyramidale, Senegalia bahiensis e Pseudobombax marginatum, com apenas um indivíduo cada, em toda a amostra.

A distribuição diamétrica dos indivíduos amostrados na área apresenta comportamento característico de populações naturais, concentrando mais indivíduos nas classes inferiores de diâmetro, caracterizando o J-invertido.

Há forte presença de espécies exóticas na área, mesmo não tendo sido amostradas altas densidades destas exóticas na mata ciliar. Contamos com a presença de Prosopis juliflora, Leucaena leucocephala, Cryptostegia madagascariensis e Malpighia glabra, espécies que naturalmente não deveriam existir na área, por ser esta uma unidade de conservação.

\section{Referências}

Alvares, C. A., Stape, J. L., Sentelhas, P. C., Gonçalves, J. L. M. \& Sparovek, G. (2013). Köppen’s climate classification map for Brazil. Meteorologische Zeitschrift, 22 (6), 711-728. 10.1127/0941-2948/2013/0507

APG - Angiosperm Phylogeny Group. (2016). An update of the Angiosperm Phylogentic Group classification for the orders and families of flowering plants: APG IV. Botanical Journal of the Linnean Society, 181(1): 1-20. https://.org/10.1111/boj.12385 
Azevedo, T., Rosa, M. R., Shimbo, J. Z., Oliveira, M. G. (2021). Relatório Anual do Desmatamento no Brasil 2020. MapBiomas, São Paulo. 93 p. < https://s3.amazonaws.com/alerta.mapbiomas.org/rad2020/RAD2020_MapBiomasAlerta_FINAL.pdf >.

Batista, F. G., Oliveira, B. T., Almeida, M. E. A., Brito, M. S., Melo, R. R. \& Alves, A. R. (2019). Florística e fitossociologia de um remanescente florestal da caatinga Caicó-RN, Brasil. Revista Desafios, 6 (3), 118-128. http://dx..org/10.20873/uftv6-7469.

BRASIL. Lei $\mathrm{n}^{\circ}$ 9.985, de 18 de julho de 2000. Regulamenta o art. 225, $\S 1^{\circ}$, incisos I, II, III, e VII da Constituição Federal, institui o Sistema Nacional de Unidades de Conservação da Natureza e dá outras providências. <http://www.camara.gov.br/sileg/integras/773285.pdf>.

Calixto Júnior, J. T. \& Drumond, M. A. (2011). Estrutura fitossociológica de um fragmento de caatinga sensu stricto 30 anos após corte raso, Petrolina-PE, Brasil. Revista Caatinga, 24 (2), 67-74.

Chaves, A. D. C. G., Santos, R. M. S., Santos, J. O., Fernandes, A. A. \& Maracajá, P. B. (2013). A importância dos levantamentos florístico e fitossociológico para a conservação e preservação das florestas. ACSA, 9(2), 43-48. http://revistas.ufcg.edu.br/acsa/index.php/ACSA/article/view/449/pdf.

CNUC - Cadastro Nacional de Unidade de Conservação do Ministério do Meio Ambiente. (2020). Unidades de Conservação por Bioma. https://antigo.mma.gov.br/images/arquivo/80229/CNUC_FEV20\%20-\%20C_Bio.pdf

Dias, B. F. S. (2001). Demandas Governamentais para o Monitoramento da Diversidade Biológica Brasileira. In: Garay, I. \& Dias, B. (Orgs) Conservação da Biodiversidade em Ecossistemas tropicais: avanços conceituais e revisão de novas metodologias de avaliação e monitoramento (pp. 17-28). Editora Vozes.

Farias, R. C., Lacerda, A. V., Gomes, A. C., Barbosa, F. M. \& Dornelas, C. S. M. (2017). Riqueza florística em uma área ciliar de Caatinga no Cariri Ocidental da Paraíba, Brasil. Revista Brasileira de Gestão Ambiental e Sustentabilidade. 4(7), 109-118. https://dx..org/10.21438/rbgas.040711

Fernandes, J. D. \& Medeiros, A. J. D. (2009). Desertificação no Nordeste: uma aproximação sobre o fenômeno do Rio Grande do Norte. HOLOS, 3(25), 147161.

Flora do Brasil (2020). Jardim Botânico do Rio de Janeiro. http://floradobrasil.jbrj.gov.br.

Giulietti, A. M., Bocage Neta, A. L., Castro, A. A. J., Rojas, C. F. L. G., Sampaio, E. V. S. B., Virgínio, J. \& Harley, R. M. (2004). Diagnóstico da vegetação nativa do bioma Caatinga. (pp. 48-90). In: Silva, J. D., Tabarelli, M., Fonseca, M. D. \& Lins, L. V. (Orgs.). Biodiversidade da Caatinga: áreas e ações prioritárias para a conservação. Brasília: Ministério do Meio Ambiente.

Guedes, R. S., Zanella, F. C. V., Costa Junior, J. E. V., Santana, G. M. \& Silva, J. A. (2012). Caracterização florístico-fitossociológica do componente lenhoso de um trecho de caatinga no semiárido paraibano. Revista Caatinga, 25(2), 99-108. https://periodicos.ufersa.edu.br/index.php/caatinga/article/view/2231.

IBGE. Instituto Brasileiro de Geografia e Estatística. Divisão regional do Brasil em regiões geográficas imediatas e regiões geográficas intermediárias. Rio de Janeiro: IBGE, 2017. 〈https://biblioteca.ibge.gov.br/visualizacao/livros/liv100600.pdf>

Justino, S. T. P., Silva, R. P. S., Freitas, A. L., Sales, F. C. V., Lima, A. A., Silva, J. L. B. L., Lima, L. V. S., Cavalcante, A. E. Q. M. \& Medeiros, F. S. (2019). Floristic and Phytoosociological Survey in a Caatinga Fragment under Extensive Grazing in Patos - PB Municipality. Journal of Experimental Agriculture International, 29(6), 1-12. 10.9734/JEAI/2019/46658.

Lacerda, A. V \&, Barbosa, F. M. (2018). Fitossociologia da vegetação arbustivo-arbórea em uma área de mata ciliar no semiárido paraibano, Brasil. Gaia Scientia, 12(2), 34-43. https://periodicos.ufpb.br/ojs2/index.php/gaia/article/view/35719/20280

Leal, I. R., Silva, J. M. C., Tabarelli, M. \& Lacher Junior, T. E. (2005). Mudando o curso da conservação da biodiversidade na Caatinga do Nordeste do Brasil. Revista Megadiversidade. 1(1), 139-146.

Lima, W. P. (2008). Hidrologia Florestal Aplicada ao Manejo de Bacias Hidrográficas (2 ed.). USP.

Lima, J. R., Silva, R. G., Tomé, M. P., Sousa Neto, E. P., Queiroz, R. T., Branco, M. S. D. \& Moro, M. F. (2019). Fitossociologia dos componentes lenhoso e herbáceo em uma área de caatinga no Cariri Paraibano, PB, Brasil. Hoehnea. 46(3), e792018. http://dx..org/10.1590/2236-8906-79/2018.

Marques, F. J., Cabral, A. G. A., Lima, C. R. \& França, P. R. C. (2020). Florística e estrutura do componente arbustivo-arbóreo da caatinga nas margens do rio Sucuru em Coxixola, Paraíba: reflexos da antropização. Braz. J. of Develop. 6(4), 20058-20072. 10.34117/bjdv6n4-252

Medeiros, F. S., Souza, M. P., Cerqueira, C. L., Alves, A. R., Souza, M. S. \& Borges, C. H. A. (2018). Florística, fitossociologia e modelagem da distribuição diamétrica em um fragmento de Caatinga em São Mamede-PB. ACSA, 14(2), 85-95. http://dx..org/10.30969/acsa.v14i2.900

Medeiros, N. C. G., Sousa, S. M. S., Monteiro, M. M., Azevedo, L. K. S. \& Sales, F. C. V. (2021). Levantamento florístico e fitossociológico em remanescente florestal em Patos-PB. Revista Desafios, 8(1), 120-129. http://dx..org/10.20873/uftv8-9968

Moreira, M. M. (2013). Estoque de carbono e nitrogênio em áreas de vegetação nativa e antropizada no município de Irecê-BA. [Dissertação de Mestrado]. Universidade Federal do Recôncavo da Bahia.

PARAÍBA (2002). Decreto n. $^{\circ} 23.832$ de 27 de dezembro de 2002. Cria o Monumento Natural Vale dos Dinossauros e dá outras providências.

Ramos, G. G., Alves, J. B., Araújo, M. F., Ferreira, V. S. G., Pinto, M. G. C., Leite, M. J. H., Vasconcelos, A. D. M. \& Ribeiro, I. R. (2020). Levantamento dos impactos ambientais de um trecho de mata ciliar em região de Caatinga no Sertão Paraibano. Braz. J. of Develop., 6(7), 52848-52859. 10.34117/bjdv6n7798.

RMFC - Rede de Manejo Florestal da Caatinga. (2005). Protocolo de Medições de Parcelas Permanentes. Associação de Plantas do Nordeste. 
Research, Society and Development, v. 10, n. 17, e39101724134, 2021

(CC BY 4.0) | ISSN 2525-3409 | DOI: http://dx.doi.org/10.33448/rsd-v10i17.24134

Rocha, J. G. Modelagem de conhecimento e métricas de paisagem para identificar e analisar padrões espaciais em ambiente de caatinga. $2011.153 \mathrm{f}$. Dissertação (Mestrado). Programa de Pós-Graduação em Ciências Geodésicas e Tecnologia da Geoinformação, Universidade Federal de Pernambuco, Recife, 2011.

Santos, W. S., Souza, M. P., Nóbrega, G. F. Q., Medeiros, F. S., Alves, A. R. \& Holanda, A. C. (2017). Caracterização florístico-fitossociológica do componente lenhoso em fragmento de caatinga no município de Upanema-RN. Nativa, 5(2), 85-91. 10.5935/2318-7670.v05n02a02.

Silva, F. G., Silva, R. H., Araújo, R. M., Lucena, M. F. A. \& Sousa, J. M. (2015). Levantamento florístico de um trecho de mata ciliar na mesorregião do Sertão Paraibano. Revista Brasileira de Biociência, 13(4), 250-258. http://www.ufrgs.br/seerbio/ojs/index.php/rbb/article/view/3163

Silva, L. S., Alves, A. R., Nunes, A. K. A., Macedo, W. S. \& Martins, A. R. (2015). Florística, estrutura e sucessão ecológica de um remanescente de mata ciliar na bacia do Rio Gurguéia-PI. Nativa, 3(3), 156-164. http://dx..org/10.14583/2318-7670.v03n03a02

Silva, A. G. da., Vilar, L. O., Vilar, V. O., Coelho, F. P., Acioli, N. R. dos S., Ramos, R. B. G. A., Moreira, J. G., Diares, T. R., Silva, D. F. da, Cruz, M. S. da, \& Moura, R. G. de. (2021). O manejo florestal sustentável da caatinga. Revista Ibero-Americana De Humanidades, Ciências e Educação, 7(5), 872-884. https://.org/10.51891/rease.v7i5.1299

Souza, G. F. \& Medeiros, J. F. (2013). Fitossociologia e Florística em áreas de caatinga na Microbacia hidrográfica do Riacho Cajazeiras - RN. Revista Biotemas, 3(1), 161-176.

Souza, A. L. \& Soares, C. P. B. (2013). Florestas nativas: estrutura, dinâmica e manejo. UFV. 